\title{
A double moral hazard model of organization design*
}

\author{
Elazar Berkovitch ${ }^{\dagger} \quad$ Ronen Israe $^{\ddagger} \quad$ Yossi Spiegel $^{\S}$ \\ IDC Herzliya IDC Herzliya Tel Aviv University \\ Current version: December 20, 2007
}

\begin{abstract}
We develop a theory of organization design in which the firm's structure is chosen to mitigate moral hazard problems in the selection and the implementation of projects. For a given set of projects, the "divisional structure" which gives each agent the full responsibility over a subset of projects is in general more efficient than the "functional structure" under which projects are implemented by teams of agents, each of whom specializes in one task. However, the ex post efficiency of the divisional structure may encourage the firm's manager to select more expensive (but still profitable) projects ex ante. We examine how the tradeoff between the ex post inefficiency in the implementation of projects and the ex ante inefficiency in the selection of projects is affected by various factors like size, complexity, and asymmetry in the importance of tasks. We also explore the desirability of adopting a narrow business strategy and the implications of managerial work overload for organizational structure.
\end{abstract}

Keywords: Divisional structure, functional structure, project selection, moral hazard, moral hazard in teams, narrow business strategy

JEL Classification Numbers: D23, L23

\footnotetext{
${ }^{*}$ For their comments we thank seminar participants in Chicago Business School, IDC Herzliya, the Technion, Tel Aviv University, and the University of Mannheim. Preliminary versions of the paper were also presented in the 2001 Economeric Society meetings in New Orleans and the 2003 SET meetings in Milano.

†The Interdisciplinary Center, Herzliya, Israel. email: elib@idc.ac.il

†The Interdisciplinary Center, Herzliya, Israel. email: risrael@idc.ac.il

${ }^{\S}$ Recanati Graduate School of Business Adminstration, Tel Aviv University, email: spiegel@post.tau.ac.il, http://www.tau.ac.il/ spiegel
} 


\section{Introduction}

We develop a theory of organization structure in which the optimal structure is chosen to mitigate moral hazard problems in the selection and the implementation of projects. For the sake of concreteness, we shall refer to the organization as a "firm," although our results apply equally well to other types of organizations, including the government, government agencies, and non-for-profit organizations. Specifically, we consider the choice between a "divisional structure" and a "functional structure." Under the divisional structure, also known as the

"M-form," the firm is organized as a collection of self-contained divisions, each of which has the full responsibility over a subset of projects, and needs to perform all tasks associated with these projects (e.g., production, marketing, finance, human resources, R\&D, etc.). Under functional structure, also known as the "U-form," the firm is organized as a collection of functional departments, each of which specializes in one task and performs it on all projects that the firm undertakes. Therefore, under the functional structure, each project is executed by a team of experts who belong to different functional departments.

To examine the advantages and disadvantages of the divisional and functional structures, we consider a firm that consists of a board of directors, a manager, and two agents (mid-level managers, business units, or simply employees). The manager's role is to select projects and recommend them to the board of directors. The board of directors either accepts or rejects the manager's recommendation. If they accept, then the two agents need to perform tasks like production and marketing on each project. In the context of this setting, the divisional structure corresponds to the case where each agent gets the full responsibility over a subset of projects and needs to perform all tasks on these projects, while the functional structure corresponds to the case where each agent specializes in one task and performs it on all selected projects.

Our main premise is that the selection of projects by the manager is subject to a moral hazard problem: the manager may prefer to recommend expensive projects which he personally likes even if there are more profitable projects around. We examine the effect of organization structure on this managerial moral hazard problem, as well as its effect on the agents' incentives when they implement the selected projects. We show that for a given set of 
selected projects, the divisional structure is more efficient ex post because it enables the firm to offer each agent an incentive contract that ties the agent's compensation directly to his performance. By contrast, under the functional form, each project requires the joint effort of two agents and hence there is now a moral hazard in teams problem (e.g., Alchian and Demsetz, 1972) as each agent has an incentive to free-ride on the effort of the other agent. While the functional structure is less efficient ex post, it may induce the manager to select more efficient projects ex ante if he anticipates that inefficient projects will be rejected by the board of directors. Hence, the optimal organization structure is determined by trading-off its effect on the ex ante selection of projects and its effect on the ex post implementation of selected projects.

Among other things, we show that relative to firms with a divisional structure, firms with a functional structure will have fewer but more profitable projects, their projects will have a smaller variance of gross returns and will be less likely to succeed, and these firms will offer their agents a lower expected compensation. We also show that the functional structure is more likely to be more profitable than the divisional structure when firms (i) operate in more competitive environments, (ii) have more expensive projects, and (iii) use technologies which exhibit weak economies of scope and strong economics of scale. In addition, we examine how the overall profitability of the divisional and functional structures changes when firms grows and can adopt more projects, when projects become more complex and require more tasks, and when the tasks have asymmetric effects on the probability that projects will succeed. We also consider the possibility that the firm will adopt a narrow business strategy in order to mitigate managerial moral hazard in the selection of projects.

The study of organization designs was pioneered by Chandler (1962) who argued that as firms like DuPont, General Motors, Sears, and Standard Oil grew and adopted more diverse product lines, the difficulties in coordinating functions across product lines induced them to switch from the functional structure (U-form) to the divisional structure (M-form). Chandler then concluded that a firm's structure follows its strategy which determines the number and type of its product lines. Our model shows that the interaction between strategy and structure can run both ways: holding fixed the firm's strategy which is determined by the manager's choice of projects, the divisional structure dominates the functional structure 
ex post. However, the functional structure may improve the manager's selection of projects ex ante. This suggests that both structure and strategy are determined simultaneously by more fundamental factors like technology (economies of scale and scope), the availability of various kinds of projects (i.e., the degree to which product lines are diverse), and the manager's ability to identify profitable projects.

Several papers have already examined the choice between the functional and the divisional structures. Like us, these papers consider a firm that produces two goods which require two tasks each. The divisional structure corresponds in this framework to the grouping of agents according to products, whereas the functional structure corresponds to the grouping of agents according to tasks. The papers differ from one another, as well as from our paper, in various details and they focus on different kinds of trade-offs that determine the choice between the two structures. Moreover, the firm's projects are given exogenously in these papers; hence, unlike in our paper, the firm's structure does not affect its strategy (i.e., the choice of projects).

Aghion and Tirole (1995) consider a model in which the functional structure requires agents to specialize in specific tasks and hence economizes on the cost of training agents, but the divisional structure enables agents to better signal their talent to the external job market and therefore provides stronger incentives to exert effort. ${ }^{1}$ They show that as managerial work overload increases, the divisional structure becomes more attractive relative to the functional structure since then, the manager relies more often on the agents' decisions and this improves their ability to signal talent to the external job market. In Rotemberg (1999), the firm can better control agents who perform the same task under the functional structure, but cross-task coordination is more efficient under the divisional structure. He shows that the divisional structure dominates the functional structure when the number of employees is sufficiently large. Qian, Roland, and Xu (2006) consider a model in which the divisional structure eliminates the need for costly cross-division communication to coordinate tasks, but the functional structure economizes on the cost of coordination by coordinating tasks

\footnotetext{
${ }^{1}$ Although the functional structure in Aghion and Tirole (1995) also gives rise to a team problem, the agents in their model do not receive monetary incentives as in our model and are motivated instead by career concerns.
} 
on a company-wide basis. The divisional structure is particularly attractive in their model when there is a need for local experimentation of uncertain innovations that involve several tasks; such experimentation is inefficient under the functional structure due to the need for costly communication among different divisions that engage in different tasks. Maskin, Qian, and $\mathrm{Xu}(2000)$ consider a model in which the functional structure exploits economies of scale by grouping similar tasks in the same division, but the divisional structure provides better incentives because it promotes yardstick competition among similar divisions. Besanko, Régibeau, and Rockett (2005) focus on the role of risk aversion: under the divisional structure, the compensation of agents depends only on their own (risky) performance, whereas under the functional structure it also depends on the (risky) performance of other agents. Hence, agents must receive a larger risk premium under the functional structure in order to induce them to exert the same level of effort. This result may be reversed however if there are significant asymmetries in the contribution of the two tasks to profits, or significant positive externalities across tasks.

Harris and Raviv (2002) consider a firm with two product lines which require two tasks each. The comparison between the divisional and functional structures depends on the likelihood that various cross-task interactions will be realized, as well as on the CEO's cost of coordinating company-wide interactions between all four tasks. For a wide range of parameters, both the functional and the divisional structures are dominated by either the matrix form whereby each task is coordinated by two different middle managers, or by a flat hierarchy whereby only the CEO may coordinate cross-task interactions. ${ }^{2}$

The rest of the paper is organized as follows. Section 2 describes the model. Section 3 characterizes the expected ex post profit of the firm under the divisional and functional structures for a given set of selected projects. In Section 4 we take the selection of projects into account and compare the overall payoff of the firm under the two structures. In Section 5 we study how the optimal structure of the firm changes when the firm grows and can

\footnotetext{
${ }^{2}$ Mookherjee and Tsumagari (2001) also consider the possibility that the firm will adopt a matrix form. The key disadvantage of the divisional structure is that it does not allow one division to source cheap components from a rival division when the latter happens to be more efficient. Although the functional structure allows for this possibility, the resulting benefits accrue disproportionately to the division managers rather than the firm's owners. The matrix form overcomes both problems and hence dominates both the functional and the divisonal structures.
} 
adopt more projects, when products become more complex and require more tasks, and when tasks have asymmetric effect on the probability that projects will succeed. In Section 6, we consider the possibility that the firm will adopt a narrow business strategy and will specialize in only one type of projects. In Section 7 we use our framework in order to examine the effect of managerial work overload on organizational structure. Concluding remarks are in Section 8.

\section{The model}

Consider a firm that consists of a board of directors, a manager, and two agents (middle managers, business units, or simply employees). The manager's task is to screen projects and recommend them to the board of directors. The board of directors either approves the manager's selection or rejects it. If the board of directors approves the manager's selection, the two agents need to implement the selected projects. If the board rejects the manager's selection, the game ends and all agents get a payoff of 0 .

Projects' selection: Using his expertise, the manager selects projects from a large pool of potential projects. All projects yield a return $R$ if they succeed and 0 if they fail. Projects differ only with respect to the required initial investment: L-type projects require a low initial investment which we normalize to 0 , while $\mathrm{H}$-type projects require a high initial investment equal to $I \in(0, R)$. We assume that the manager can always discover H-type projects, but can discover (at least two) L-type projects only with probability $\alpha$; with probability $1-\alpha$, the manager discovers only H-type projects.

A key assumption in our model is that the manager prefers H-type projects over Ltype projects. This preference could be due to several reasons. First, the manager may have "empire-building" preferences and may like to manage more expensive, "larger" projects. Second, it is possible that H-type projects enhance the manager's general human capital whereas L-type projects only contribute to the his firm-specific human capital. Third, it may be the case that L-type projects are "traditional," whereas H-type projects are more "innovative" and involve "cutting edge" technologies that the manager likes. For instance, a founder of a biotechnology firm may prefer projects that bring him a greater recognition in 
the scientific community even if they are more costly for the firm. Fourth, if the manager is also an entrepreneur, then he might be personally involved with the development of H-type projects, and hence may resist replacing them with more profitable L-type projects if they become available. Finally, it might be that the manager gets the same private benefit from H-type and from L-type projects but needs to exert effort in order to discover projects; since H-type projects can be discovered for sure while L-type projects are only discovered with probability $\alpha$, the manager will prefer H-type over L-type projects.

We assume that the board of directors is a perfect agent of outside investors and is interested in maximizing the net expected profit of the firm. Hence, unlike the manager, the board of directors prefers L-type projects over H-type projects. To focus on the incentive role of organization design, we assume that monetary incentives alone (any equity stake the manager has plus wages and bonuses) are insufficient to induce the manager to recommend L-type projects. Given this assumption, the manager's wage will therefore be constant and equal to his reservation wage which we normalize to zero.

We also assume that when the manager recommends H-type projects, the board of directors cannot discern whether he failed to discover L-type projects, or whether he did discover them but conceals this fact. Since the board is interested in maximizing the expected profit of the firm, it will approve the manager's recommendation only if the net expected value of the selected projects is positive. Otherwise, the board will reject the manager's recommendation and the firm will not adopt any projects. This implies in turn that whenever the net expected value of H-type projects is positive, there is a managerial moral hazard problem in the selection of projects: the manager anticipates that the board of directors will approve H-type projects and hence he recommends them even if he manages to discover more profitable L-type projects. But if the net expected value of H-type projects is negative, then there is no similar problem because now the manager anticipates that the board will reject H-type projects and hence he does not recommend them.

Projects' implementation: Once projects have been approved by the board of directors, the two agents need to perform two tasks on each project to enhance its chance to succeed. For concreteness, we refer to the two tasks as production $(\mathrm{p})$ and marketing $(\mathrm{m})$, but they 
could equally well be engineering and product design, R\&D and financing, or purchasing and sales. We assume that each agent can perform at most two tasks. Since each project also requires two tasks, the firm can at most adopt two projects. Given the efforts that the agents exert in task $\mathrm{p}, e_{p}$, and task $m, e_{m}$, the probability that a project will succeed is

$$
q\left(e_{p}, e_{m}\right)=e_{p}+e_{m}
$$

The organizational structure: We postulate that the organizational structure of the firm can mitigate, at least in part, the potential moral hazard problem in the selection of projects. Given our setup, there are two ways to allocate the two projects and the two tasks between the two agents.

One alternative is to assign each project to one agent and let each agent perform both tasks on his assigned project. We refer to this alternative as the "multidivisional structure," (i.e., the M-form) or "divisional structure" for short. Under this structure the firm essentially has two divisions, each of which is fully responsible for one project. We assume that agent $j$ 's cost of implementing his project is

$$
C^{d}\left(e_{p j}, e_{m j}\right)=\gamma e_{p j}^{2}+\gamma e_{m j}^{2}-\frac{\rho e_{p j} e_{m j}}{2}, \quad j=1,2
$$

where $e_{p j}$ and $e_{m j}$ are the efforts of agent $j$ in production and in marketing, $\gamma \geq 1$, and $\rho \in(-1,1)$ is a parameter that measures the complementarity between the two tasks. If $0<\rho<1$, then there are economies of scope and performing one task lowers the cost of performing the other task (e.g., when the agent engages in marketing he learns about the customers needs and hence can design the product more efficiently; alternatively, asking each agent to perform two different tasks increases the agents' satisfaction and self-interest and hence lowers their disutility from effort). If $-1<\rho<0$, there are diseconomies of scope and hence performing one task increases the cost of performing the other task.

A second alternative is the "Unitary functional structure," (i.e., the U-form), or "functional structure" for short. Here each agent specializes in one task and performs it on both projects. One can now think of the two agents as the "production department" and the 
"marketing department." The cost that agent $i$ incurs when performing task $i$ on projects 1 and 2 is

$$
C^{f}\left(e_{i 1}, e_{i 2}\right)=\gamma e_{i 1}^{2}+\gamma e_{i 2}^{2}-\frac{\sigma e_{i 1} e_{i 2}}{2}, \quad i=p, m
$$

where $e_{i 1}$ and $e_{i 2}$ are the efforts of agent $i$ in projects 1 and 2 and $\sigma \in(-1,1)$ is a parameter that measures the degree of economies of scale if $0<\sigma<1$ or diseconomies of scale if $-1<\sigma<0$. In other words, when $\sigma>0(\sigma<0)$, performing each task twice lowers (increases) the cost of effort.

The two organizational structures are illustrated in Figure 1.

Divisional structure

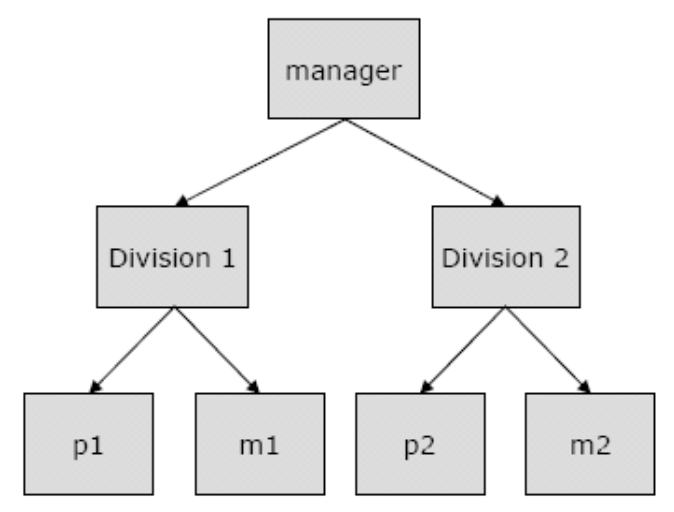

Functional structure

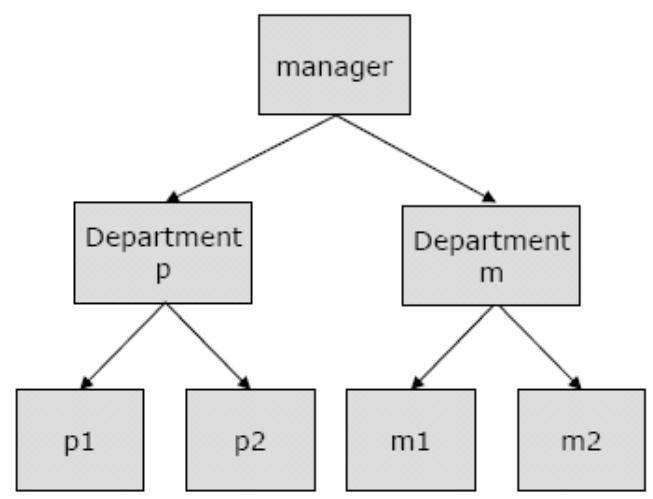

Figure 1: Illustrating the divisional and the functional structures

The timing: First, the board of directors sets up the organizational structure of the firm. Then, the manager screens projects and recommends two of them to the board of directors. The board in turn either accepts the manager's recommendation or rejects it. In the latter case, no projects are implemented and all agents get a payoff of 0 . If the board accepts the manager's recommendation, it signs incentive contracts with the two agents. Finally, the agents implement the selected projects and payoffs are realized.

Note that we assume that the board, not the manager, signs contracts with the agents. The logic behind this assumption is that after projects are selected, the manager's incentives are not fully aligned with those of the board. In particular, if the manager gets 
extra private benefits when projects succeed, then he may wish to maximize the probability of success rather than profits. Moreover, we assume that the board sets up the organizational structure before projects are selected, but signs contracts with the agents after projects are selected. This reflects the idea that organizational structure is a long-term decision while incentive contracts are a more short-term decision which is easier to modify. Hence, although the board can offer the agents contracts at the outset and design them so as to motivates the manager to select L-type, the board will have an incentive to renegotiate these contracts

once the manager has recommended projects in order to ensure that the selected projects are implemented efficiently.

It is also worth noting that if the manager is averse to firm's losses (say because his reputation is damaged in this case), then he will not select H-type projects when they are not profitable. In this case, the formal approval of projects by the board of directors is not needed in order to mitigate managerial moral hazard in the selection of projects.

\section{The expected ex post profit of the firm under the two organizational structures}

In this section we compute the expected ex post profit of the firm (gross of the cost of investment) under the divisional and the functional structures, holding fixed the type of projects that the firm adopts. Our analysis here is therefore done from an ex post perspective. In the next section we will also take the selection of projects into account and characterize the conditions under which each organizational structure is more efficient overall.

\subsection{The divisional structure}

The salient feature of the divisional structure is that the firm is organized around projects: each project is assigned to one agent who gets the full responsibility for this project. Since there is no interaction between the two agents in this case, the board of directors can sign a contract with each agent separately.

We assume that the efforts of the two agents when they implement projects are 
nonverifiable. Hence the firm can condition the agents' compensation only on whether their respective projects succeeded or not. Let $W_{j}$ be the compensation of agent $j(j=1,2)$ if project $j$ succeeds. If no project is assigned to agent $j$, the agent gets no monetary compensation. The contract offered to agent $j$ is characterized by the solution to the following problem:

$$
\begin{aligned}
& \max _{\left\{e_{p i}, e_{m i}, W_{i}\right\}} \quad q_{1}\left(e_{p 1}, e_{m 1}\right)\left(R-W_{1}\right)+q_{2}\left(e_{p 2}, e_{m 2}\right)\left(R-W_{2}\right) \\
& \text { s.t. } \quad e_{p i}, e_{m i} \in \underset{\widehat{e}_{p i}, \widehat{e}_{m i}}{\operatorname{Arg} \max } \quad q_{i}\left(\widehat{e}_{p i}, \widehat{e}_{m i}\right) W_{1}-C^{d}\left(\widehat{e}_{p i}, \widehat{e}_{m i}\right), \quad i=1,2 \text {, } \\
& q_{i}\left(e_{p i}, e_{m i}\right) W_{i}-C^{d}\left(e_{p i}, e_{m i}\right) \geq 0, \quad i=1,2 .
\end{aligned}
$$

Solving this problem, the optimal contracts are such that

$$
W_{j}^{d}=\frac{R}{2}, \quad j=1,2 .
$$

At the optimum, the participation constraints of the two agents are non-binding, ${ }^{3}$ and the probability that project $j$ succeeds is:

$$
q_{j}^{d}=\frac{2 R}{4 \gamma-\rho}, \quad j=1,2
$$

Since $\gamma \geq 1$ and $\rho<1, q_{j}^{d}>0$. In order to avoid uninteresting technical complications, we shall assume that $\gamma$ is sufficiently large relative to $R$ to ensure that $q_{j}^{d}<1$ for all $\rho \in(-1,1)$.

Noting that the expected ex post profit from project $j$ is $q_{i}\left(e_{p i}, e_{m i}\right)\left(R-W_{i}\right)$ and using (3) and (4), it follows that the expected ex post profit per project (gross of the cost of investment) under a divisional structure is

$$
\pi^{d}=\frac{R^{2}}{4 \gamma-\rho}
$$

Equation (5) implies that $\pi^{d}$ increases with $\rho$ : the firm is more profitable ex post if there are stronger economies of scope. This is because economies of scope lower the agents' costs of

\footnotetext{
${ }^{3}$ Given $e_{p j}^{d}, e_{m j}^{d}$, and $W_{j}^{d}$, the expected payoff of agent $j$ is $\frac{R^{2}}{2(4 \gamma-\rho)}>0$.

${ }^{4}$ Formally, this assumption requires that $\gamma>\frac{2 R+\rho}{4}$.
} 
effort, so a smaller compensation is needed to induce them to exert the same level of effort.

\subsection{The functional structure}

Under the functional structure, each agent specializes in one task and performs it on both projects. Hence, each project requires the joint effort of the two agents.

As before, the agents' rewards can depend only on the success or failure of the two projects. Let $W_{i j}$ be the compensation of agent $i(i=p, m)$ if project $j(j=1,2)$ succeeds. Now, the contracts offered to the two agents are chosen to solve the following problem:

$$
\begin{aligned}
& \max \quad q_{1}\left(e_{p 1}, e_{m 1}\right)\left(R-W_{p 1}-W_{m 1}\right)+q_{2}\left(e_{p 2}, e_{m 2}\right)\left(R-W_{p 2}-W_{m 2}\right) \\
& \left\{e_{i j}, W_{i j}\right\} \\
& \text { s.t. } \quad e_{p 1}, e_{p 2} \in \underset{\widehat{e}_{p 1}, \widehat{e}_{p 2}}{\operatorname{Arg} \max } \quad q_{1}\left(\widehat{e}_{p 1}, e_{m 1}\right) W_{p 1}+q_{2}\left(\widehat{e}_{p 2}, e_{m 2}\right) W_{p 2}-C^{f}\left(\widehat{e}_{p 1}, \widehat{e}_{p 2}\right) \\
& e_{m 1}, e_{m 2} \in \underset{\widehat{e}_{m 1}, \widehat{e}_{m 2}}{\operatorname{Arg} \max } q_{1}\left(e_{p 1}, \widehat{e}_{m 1}\right) W_{m 1}+q_{2}\left(e_{p 2}, \widehat{e}_{m 2}\right) W_{m 2}-C^{f}\left(\widehat{e}_{m 1}, \widehat{e}_{m 2}\right) \\
& q_{1}\left(e_{p 1}, e_{m 1}\right) W_{p 1}+q_{2}\left(e_{p 2}, e_{m 2}\right) W_{p 2}-C^{f}\left(e_{p 1}, e_{p 2}\right) \geq 0 \\
& q_{1}\left(e_{p 1}, e_{m 1}\right) W_{m 1}+q_{2}\left(e_{p 2}, e_{m 2}\right) W_{m 2}-C^{f}\left(e_{m 1}, e_{m 2}\right) \geq 0 \text {. }
\end{aligned}
$$

Solving this problem, the optimal contracts are such that

$$
W_{p 1}+W_{m 1}=W_{p 2}+W_{m 2}=\frac{R}{2}
$$

At the optimum, the participation constraints of the two agents are non-binding, ${ }^{5}$ and the probability that project $j$ succeeds is:

$$
q_{j}^{f}=\frac{R}{4 \gamma-\sigma}, \quad j=1,2
$$

Since $\gamma \geq 1$ and $\sigma<1, q_{j}^{f}>0$. As before, we shall assume that $\gamma$ is sufficiently large relative to $R$ to ensure that $q_{j}^{f}<1$ for all $\sigma \in(-1,1){ }^{6}$

Using (6) and (7), the expected ex post per-project profit of the firm (gross of the

\footnotetext{
${ }^{5}$ Given $e_{p j}^{f}, e_{m j}^{f}$, and $W_{j}^{f}$, the expected payoff of agent $j$ is $\frac{3 R^{2}}{8(4 \gamma-\sigma)}>0$.

${ }^{6}$ Formally, we require that $\gamma>\frac{R+\sigma}{4}$.
} 
cost of investment) is given by:

$$
\pi^{f}=\frac{R^{2}}{2(4 \gamma-\sigma)}
$$

Equation (8) implies that $\pi^{f}$ increases with $\sigma$ so economies of scale make the firm more profitable. The reason is that scale economies lower the agents' cost of effort, so a smaller compensation is needed to induce the agents to exert the same level of effort.

\subsection{Comparing the expected ex post profits under the divisional and functional structures}

Using equations (5) and (8), we establish the following result:

Lemma 1: $\pi^{d}>\pi^{f}$ for all $\rho \in(-1,1)$ and all $\sigma \in(-1,1)$.

Lemma 1 implies that holding fixed the type of projects that the firm adopts, the divisional structure yields a higher expected profit than the functional structure. The intuition is that under the functional structure, the agents' compensation depends on their joint effort, so the agents have an incentive to free-ride on one another's effort; hence, we have a "moral hazard in teams" problem. Under the divisional structure, there is no such problem since the success of each project depends on the effort of only one agent. ${ }^{7}$ Notice though from equations (5) and (8) that the advantage of the divisional structure over the functional structure ex post increases with $\rho$ and decreases with $\sigma$ : economies of scope increase the ex post advantage of the divisional structure while economies of scale decrease this advantage.

Since Lemma 1 abstracts from the cost of investment and from the manager's selection of projects, it does not fully answer the question which structure is more efficient overall. In the next section we address this question.

\footnotetext{
${ }^{7}$ As mentioned in the Introduction, there is also a team problem in Aghion and Tirole (1995). However, they do not consider monetary compensation, and moreover they do not consider the effect of organization structure on the ex ante selection of projects which is a main focus of our paper.
} 


\section{The optimal organizational structure under manage- rial moral hazard}

In this section we take into account the effect of organizational structure on the ex ante selection of projects. To this end, recall that when the manager recommends two (costly) $\mathrm{H}-$ type projects, the board of directors cannot tell whether this is because he failed to discover L-type projects or because he did discover such projects but chose to conceal this fact. The manager, who gets larger private benefits when H-type projects are adopted, will therefore recommend H-type projects so long as he expects the board to approve his recommendation. Recalling that $\mathrm{H}$-type projects require an initial investment $I$, the board of directors will accept such projects under structure $s(s=d, f)$ if and only if $I \leq \pi^{s}$. On the other hand, since we normalized the cost of L-type projects to 0 , the board will always accept L-type projects if the manager recommends them. This implies in turn that the firm overcomes the managerial moral hazard problem in projects selection if $I>\pi^{s}$.

Anticipating the board's behavior, the manager will recommend costly H-type projects if and only if $I \leq \pi^{s}$; the firm's per-project profit in this case is $\pi^{s}-I$. If $I>\pi^{s}$, the manager, who prefers that the firm will adopt L-type projects rather than not adopt any projects, will recommend L-type projects if he succeeds to discover them and will not recommend any projects otherwise (his recommendation will be rejected anyway). Since the manager succeeds to discover L-type projects only with probability $\alpha$, the expected per-project profit will be $\alpha \pi^{s}(R)$. The discussion implies the following result:

Proposition 1: The optimal organizational structure of the firm is as follows:

(i) If $I<\pi^{f}$ or $I>\pi^{d}$ (both organizational structures or neither structure feature managerial moral hazard problem), then the manager recommends the same type of projects under both structures, so the divisional structure which is ex post efficient is also more profitable overall. The firm will end up adopting two H-type projects with probability 1 if $I<\pi^{f}$ and two L-type projects with probability $\alpha$ if $I>\pi^{d}$.

(ii) If $\pi^{f} \leq I \leq \pi^{d}$ (only the divisional structure features managerial moral hazard problem), the firm faces a trade-off between ex ante efficiency in the selection of projects and ex 
post efficiency in the implementation of selected projects. If $\alpha<\widehat{\alpha} \equiv \frac{\pi^{d}-I}{\pi^{f}}$, then the divisional structure is more profitable overall while if $\alpha>\widehat{\alpha}$, then the functional structure is more profitable overall. Under the divisional structure, the firm will adopt two H-type projects with probability 1, whereas under the functional structure it will adopt two L-type projects with probability $\alpha$ and no projects otherwise.

Proof: (i) If both structures or if neither structure feature managerial moral hazard problem, then organizational structure does not affect the manager's choice of projects, so the firm will adopt the ex post efficient divisional structure.

(ii) If only the divisional structure features managerial moral hazard problem, the firm faces a trade off between ex ante efficiency in the selection of projects and ex post efficiency in the implementation of selected projects. Under the divisional structure, the manager recommends two L-type projects with probability $\alpha$ and nothing with probability $1-\alpha$. Since L-type projects require no initial investment, the expected per-project profit is $\alpha \pi^{f}$. Under the functional structure, the manager recommends two H-type projects that are approved so the expected per-project profit is $\pi^{d}-I$. The result follows by comparing $\alpha \pi^{f}$ and $\pi^{d}-I$.

Proposition 1 is illustrated in Figure 2 which shows for each pair of $I$ and $\alpha$ which organization structure dominates and which kind of projects will the firm adopt.

Proposition 1 has several interesting implications. First, $\widehat{\alpha}$ falls with $I$ which is the cost of H-type projects. Consequently, the functional structure (which mitigates the managerial moral hazard problem) becomes optimal for a larger set of parameters. Intuitively, when $I$ is large, the managerial moral hazard problem which leads to the adoption of $\mathrm{H}$ type projects becomes more costly, so the firm finds it optimal to overcome this problem by adopting the ex post inefficient functional structure for a larger set of parameters.

Second, using equations (5) and (8) recalling that $\gamma \geq 1$ and $\rho<1$, it follows that $\frac{\partial \widehat{\alpha}}{\partial R}=\frac{2 I}{R \pi^{f}}>0$. Hence, holding $I$ constant, an increase in $R$ shifts $\widehat{\alpha}$ upward, so the functional structure becomes optimal for a smaller set of parameters. Intuitively, as $R$ increases, the gap between the profitability of L-type and H-type projects shrinks and hence the advantage 


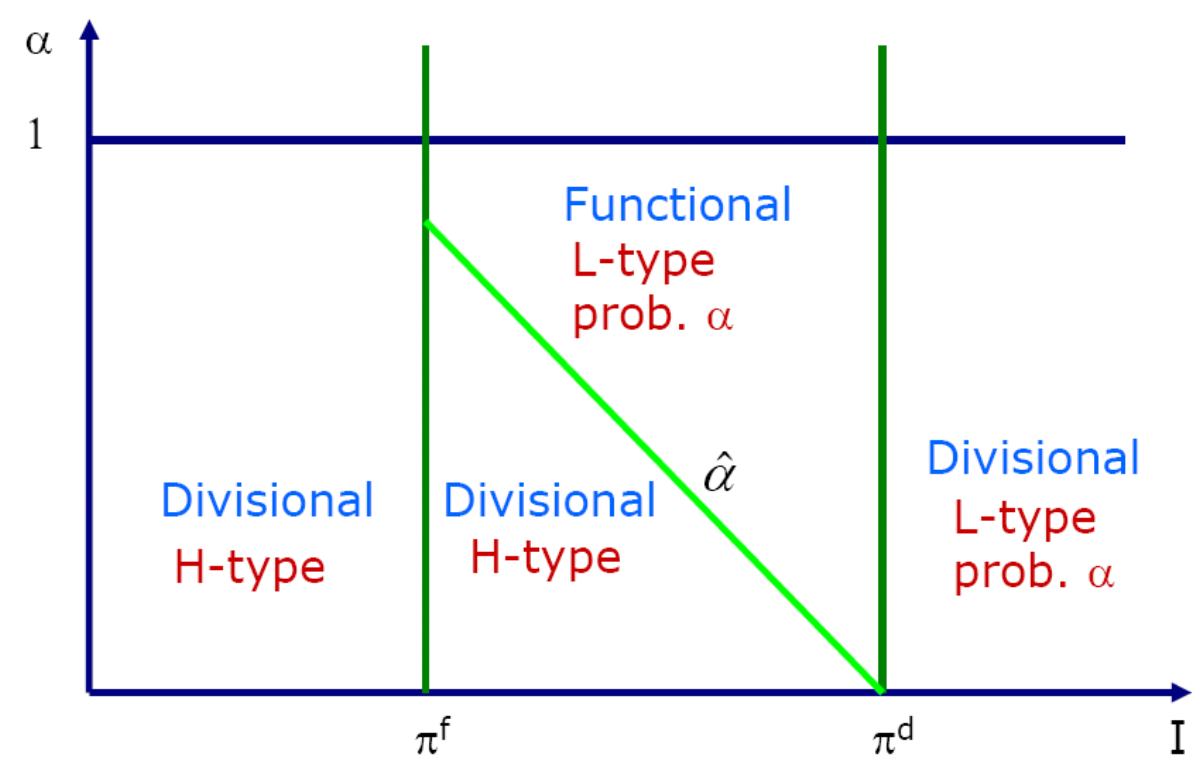

Figure 2: Comparing the divisional and the functional structures

of the functional structure (which induces the manager to adopt L-type projects) becomes smaller. If we interpret an increase in $R$ as reflecting less competition, this result suggests that firms which operate in less competitive market will be more inclined to adopt the divisional structure than firms which operate in more competitive markets.

Third, using equations (5) and (8) again, reveals that $\widehat{\alpha}$ shifts upward when $\sigma$ decreases and when $\rho$ increases. Hence, even when the managerial moral hazard problem is taken into account, economies of scale and diseconomies of scope favor the functional structure, whereas diseconomies of scale and economies of scope favor the divisional structure.

We summarize these observations in the following proposition.

Proposition 2: Suppose that $\pi^{f} \leq I \leq \pi^{d}$. Then, all else equal, the functional structure becomes optimal for a wider set of parameters as I increases (H-type projects are more costly), as $R$ decreases (the firm operates in a more competitive environment), as $\rho$ decreases (there are weaker economies of scope), and as $\sigma$ increases (there are stronger economics of scale).

We now proceed by conducting the following thought experiment. Suppose that we take a sample of firms that differ only with respect to $I$ and $R$ and divide it into two subsamples: one with firms that have a divisional structure and one with firms that have a 
functional structure. Then, on the basis of Proposition 1, what kind of differences should we expect to see between the two subsamples?

First, recall from Proposition 1 that under the divisional structure, the firm always adopts projects, but under the functional structure it adopts projects only with probability $\alpha$. Hence, firms with a divisional structure should have more projects than firms with a functional structure. Moreover, since the firm adopts only H-type projects under the divisional structure and only L-type projects under the functional structure, the per-project profit is $\pi^{d}-I$ under the divisional structure and $\pi^{f}$ under the functional structure. Since the functional structure is adopted whenever $\alpha \pi^{f} \geq \pi^{d}-I$, it follows that for firms with a functional structure, $\pi^{f}>\pi^{d}-I$. That is, the profitability of adopted projects is higher under the functional structure. On the other hand, since $\gamma \geq 1, \rho<1$, and $\sigma<1$, it follows from equations (4) and (7) that $q^{d}>q^{f}$ : projects under the functional structure are more likely to succeed than projects under the divisional structure.

Second, recall that the two agents receive monetary compensation only when projects are adopted and succeed. Using equations (3), (4), (6), and (7), the expected compensation of the agents is equal to $\pi^{d}$ under the divisional structure and $\alpha \pi^{f}$ under the functional structure. Since $\alpha \leq 1$ and given Lemma 1, it is clear that the expected compensation is lower under the functional structure.

Third, note that Proposition 1 predicts that a necessary (but not sufficient) condition for the functional structure to be optimal is that $\pi^{f} \leq I \leq \pi^{d}$; using (8) and (5), this condition can be written as $\sqrt{(4 \gamma-\rho) I} \leq R \leq \sqrt{2(4 \gamma-\sigma) I}$. By contrast, the divisional structure can be optimal for all values of $R$ : when $R<\sqrt{(4 \gamma-\rho) I}$ or $R>\sqrt{2(4 \gamma-\sigma) I}$, the divisional structure is always optimal, and when $\sqrt{(4 \gamma-\rho) I} \leq R \leq \sqrt{2(4 \gamma-\sigma) I}$ it is optimal provided that $\alpha$ is sufficiently low. Hence, if we fix $I$ and assume that $R$ is drawn from some distribution on the support $\left[R_{0}, R_{1}\right]$, where $R_{0}<\sqrt{(4 \gamma-\rho) I}$ and $R_{1}>\sqrt{2(4 \gamma-\sigma) I}$, then the variance of gross returns (excluding the cost of investment) will be larger under the divisional structure than under the functional structure.

Proposition 3: Relative to firms with a divisional structure, firms with a functional structure 
(i) have fewer but more profitable projects,

(ii) have projects that are less likely to succeed,

(iii) pay a lower expected compensation to their agents,

(iv) have projects with a smaller variance of gross returns.

The main insight in this section is that the ex post inefficient functional structure can mitigate the manager's incentive to recommend H-type projects. ${ }^{8}$ A natural question to ask is whether the firm can induce the manager to select L-type projects by other means. Although we assume that monetary incentives alone are insufficient for that purpose, the board of directors may still be able to induce the manager to select L-type projects by threatening to fire him if he recommends H-type projects. However, if the manager is needed in order to complete the projects that he selected (say because he has some inalienable human capital that is an important input in the execution of projects), then firing him would hurt the firm's performance and hence be worse than adopting a functional structure. Another possibility is that the board will commit the firm to focus on L-type projects by imposing a technological constraint on the firm's ability to implement H-type projects. We consider this possibility in detail in Section 6. One more possibility is to distort the agents' compensation when H-type projects are selected such that these projects will be unprofitable. But, as we discussed earlier, such a scheme may not be credible because the board of directors will have an incentive to renegotiate the agents' contracts once the manager has recommended H-type projects in order to ensure that they are implemented efficiently. Anticipating this incentive, the manager will not be deterred from recommending H-type projects.

\section{Extensions}

In this section we study how our model can shed light on various questions of interest such as how the profitability of the two organizational structures changes when the firm grows and

\footnotetext{
${ }^{8}$ Note that if the manager is also the founder of the firm, then he may wish to set up the functional structure himself in order to credibly commit to outside investors that he will not select inefficient projects.
} 
can adopt more projects, or when projects become more complex and require more tasks, or when the tasks have asymmetric effects on the probability that projects will succeed.

\subsection{The firm can adopt $2 n$ projects}

Suppose that for some exogenous reason, each agent can complete $2 n$ tasks, where $n$ is a positive integer. Given this assumption, the firm can adopt $2 n$ projects: under the divisional structure, it will assign $n$ projects to each agent who will then perform tasks $p$ and $m$ on $n$ projects (thus completing a total of $n$ tasks). Under the functional structure, each agent will specialize in one task and will perform it on all $2 n$ projects. To simplify matters, we will assume that with probability $\alpha$ there are at least $2 n$ L-type projects and with probability $1-\alpha$, there are no L-type projects available. As before, this assumption will ensure that all the projects that the firm adopts are of the same type. To avoid uninteresting technical complications, we shall assume that $n<\frac{4 \gamma+1}{2}$.

Let $N_{1}=\{1, \ldots, n\}$ and $N_{2}=\{n+1, \ldots, 2 n\}$ be sets of projects that are assigned to agents 1 and 2, respectively. Under the divisional structure, agent $j$ 's cost of effort becomes

$$
C_{j}^{d}=\gamma \sum_{\ell \in N_{j}}\left(e_{p \ell}^{2}+e_{m \ell}^{2}\right)-\frac{\rho}{2} \sum_{\ell \in N_{j}} e_{p \ell} e_{m \ell}-\frac{\sigma}{2} \sum_{\ell \in N_{j}} \sum_{\ell^{\prime} \in N_{j} \backslash \ell}\left(\frac{e_{p \ell} e_{p \ell^{\prime}}+e_{m \ell} e_{m \ell^{\prime}}}{2}\right), \quad j=1,2
$$

Under the functional structure, each agent specializes in one task and preforms it $n$ times. Consequently, agent $i$ 's cost of effort is given by

$$
C_{i}^{f}=\gamma \sum_{\ell=1}^{2 n} e_{i \ell}^{2}-\frac{\sigma}{4} \sum_{\ell=1}^{2 n} \sum_{\ell^{\prime}=1, \ell^{\prime} \neq \ell}^{2 n}\left(\frac{e_{i \ell} e_{i \ell^{\prime}}}{2}\right), \quad i=p, m
$$

When $n=1, N_{1}$ and $N_{2}$ are singletons (i.e., each agent gets the full responsibility for only one project), so $C_{j}^{d}$ coincides with the cost function in equation (1). Hence equation (9) generalizes the cost function in equation (1) to the case where $n>1$. Likewise, when $n=1$, equation (10) coincides with equation (2). Note that since under the divisional structure each agent needs to perform tasks $p$ and $m$ on more than one project, $C_{j}^{d}$ is now affected not

only by economies of scope but also by economies of scale. By contrast, $C_{i}^{f}$ is affected only by economies of scale because, as before, each agent specializes in only one task. 
Assuming that the firm adopts $n$ identical projects, it is straightforward to verify that the expected ex post profit per-project under the divisional structure is given by:

$$
\pi^{d}(n)=\frac{R^{2}}{4 \gamma-\rho-(n-1) \sigma}
$$

whereas under the functional structure, it is given by:

$$
\pi^{f}(n)=\frac{R^{2}}{2(4 \gamma-(2 n-1) \sigma)}
$$

The assumption that $n<\frac{4 \gamma+1}{2}$ ensures that the numerators of (11) and (12) are positive. When $n=1, \pi^{d}(n)$ and $\pi^{f}(n)$ coincide with the expressions in (5) and (8). Hence, equations (11) and (12) generalize the previous analysis to the case where $n>1$. Notice that $\pi^{d}(n)$ increases with both $\rho$ and $\sigma$. The reason why $\pi^{d}(n)$ is affected not only by economies/diseconomies of scope as in the case of $n=1$, but also by economies/diseconomies of scale is that now each agent repeats each task $n$ times. Likewise, it is easy to see that $\pi^{f}(n)$ increases with $\sigma$ at an increasing rate.

In order to examine how $n$ affects the optimal organizational structure, note first that both $\pi^{d}(n)$ and $\pi^{f}(n)$ are increasing with $n$ if $\sigma>0$ but are decreasing with $n$ if $\sigma<0$. Recalling that managerial moral hazard arises when the expected ex post profit per project exceeds $I$ (the manager anticipates that the board will approve H-type projects), it follows that an increase in $n$ makes the managerial moral hazard problem more severe if $\sigma>0$ (there are economies of scale) but less severe if $\sigma<0$ (there are diseconomies of scale).

Next, using equations (11) and (12) we obtain

$$
\frac{\partial}{\partial n}\left(\frac{\pi^{d}(n)}{\pi^{f}(n)}\right)=\frac{-2 \sigma(4 \gamma-2 \rho+\sigma)}{(4 \gamma-\rho-(n-1) \sigma)^{2}}<0
$$

Hence, an increase in $n$ pushes $\pi^{f}(n)$ and $\pi^{d}(n)$ closer together if $\sigma>0$ (there are economies of scale) but further away from each other if $\sigma<0$ (there are diseconomies of scale). Moreover, note that

$$
\operatorname{sign}\left[\pi^{d}(n)-\pi^{f}(n)\right]=\operatorname{sign}[4 \gamma+\rho-(3 n-1) \sigma] .
$$


This expression is surely positive if $\sigma<0$, but could be negative if $\sigma>0$ and if $n$ is sufficiently large. To illustrate, suppose that $\sigma=1, \rho=0$, and $\gamma=5$. Then, $4 \gamma+\rho-(3 n-1) \sigma=21-3 n$,

so $\pi^{d} \geq \pi^{f}$ if $n \leq 7$ and $\pi^{d}<\pi^{f}$ if $n \geq 8$. Since by assumption $n<\frac{4 \gamma+1}{2}=10.5$, it follows that $\pi^{d}(n)<\pi^{f}(n)$ when $n=8,9,10$.

Proposition 4: An increase in $n$ has the following effects:

(i) It makes the managerial moral hazard problem more severe if $\sigma>0$ (there are economies of scale) but less severe if $\sigma<0$ (there are diseconomies of scale).

(ii) It favors the functional structure in the sense that it lowers $\frac{\pi^{d}(n)}{\pi^{f}(n)}$ if $\sigma>0$ and favours the divisional structure if $\sigma<0$. As $n$ becomes sufficiently large, $\pi^{f}(n)$ may exceed $\pi^{d}(n)$ provided that $\sigma$ is positive and sufficiently large.

Intuitively, although an increases in $n$ means that economies/diseconomies play a more significant role under both structures, each agent performs each task $n$ times under the divisional structure but $2 n$ times under the functional structure. Hence, economies/diseconomies of scale affect the functional structure more than they affect the divisional structure. Consequently, an increase in $n$ favors the functional structure if there are economies of scale, but favors the divisional structure if there are diseconomies of scale.

\subsection{Each project requires $2 k$ tasks}

We now wish to examine how the optimal structure of the firm is affected by the complexity of the projects which the firm adopts. To this end, we shall identify complexity with the number of tasks that each project requires and will assume that each project requires $2 k$ tasks, where $k$ is a positive integer (in total then the firm as a whole needs to perform $4 k$ tasks). In order to maintain the feature that the firm has the capacity to adopt only two projects, we will assume in addition that there are $2 k$ agents. As before, each agent can perform only two tasks. To ensure that the probability that a project will succeed does not increase simply due to the increase in the number of tasks, we divide the sum of the efforts 
by $k$ :

$$
q\left(e_{1}, \ldots, e_{2 k}\right)=\sum_{j=1}^{2 k} \frac{e_{j}}{k} .
$$

This modification ensures that if each agent exert the same effort as in Section 3, then the likelihood of success will be exactly as in Section 3. Consequently, an increase in $k$ will affect matters only through its effect on the agents' incentives.

Given that the firm can adopt at most two projects, it is clear that under the divisional structure the firm will establish two divisions and will assign each division the full responsibility over one project. However, unlike before, now there are $k \geq 1$ agents in each division who need to split $2 k$ tasks among themselves. Naturally then, this situation will give rise to a moral hazard in teams problem. The difference though between this case and the functional structure studied earlier is that here each agent performs two different tasks on the same project whereas under the functional structure, each agent performs the same task on two different projects.

Using $A_{i}$ and $B_{i}$ to denote the tasks assigned to agent $i$, we can write the cost that each agent $i$ incurs under the divisional structure as

$$
C^{d}\left(e_{i A_{i}}, e_{i B_{i}}\right)=\gamma e_{i A_{i}}^{2}+\gamma e_{i B_{i}}^{2}-\frac{\rho e_{i A_{i}} e_{i B_{i}}}{2}
$$

As before, the cost of effort under the divisional structure is affected by economies/diseconomies of scope since each agent performs two different tasks.

Since the two projects are completely independent, we can characterize the optimal contracts by considering the firm's problem vis-à-vis one division (the problem vis-à-vis the other division is completely identical). Since we use $A_{i}$ and $B_{i}$ to denote the tasks assigned to agent $i$, the probability that the project that was assigned to the division succeeds can be written as $\sum_{i=1}^{k} \frac{\left(e_{i A_{i}}+e_{i B_{i}}\right)}{k}$. The contracts that the firm offers to the $k$ agents who work in 
the division are characterized by the solution to the following problem:

$$
\begin{aligned}
& \max \\
& \left\{e_{i A_{i}}, e_{i B_{i}}, W_{i}\right\} \\
& \sum_{j=1}^{k} \frac{\left(e_{i A_{i}}+e_{i B_{i}}\right)}{k}\left(R-\sum_{i=1}^{k} W_{i}\right) \\
& \text { s.t. } \quad e_{i A_{i}}, e_{i B_{i}} \in \underset{\widehat{e}_{i A_{i}} \widehat{e}_{i B_{i}}}{\operatorname{Arg} \max }\left(\frac{\widehat{e}_{i A_{i}}+\widehat{e}_{i B_{i}}}{k}+\sum_{j \neq i}^{k} \frac{\left(e_{j A_{i}}+e_{j B_{i}}\right)}{k}\right) W_{i}-C^{d}\left(\widehat{e}_{i A_{i}}, \widehat{e}_{i B_{i}}\right), \quad i=1, \ldots, k \text {, } \\
& \left(\sum_{i=1}^{k} \frac{\left(e_{i A_{i}}+e_{i B_{i}}\right)}{k}\right) W_{i}-C^{d}\left(e_{i A_{i}}, e_{i B_{i}}\right) \geq 0, \quad i=1, \ldots, k .
\end{aligned}
$$

Solving this problem reveals that under a divisional structure, the expected ex post per-project profit (gross of the cost of investment) is

$$
\pi^{d}(k)=\frac{\pi^{d}}{k^{2}}
$$

where $\pi^{d}$ is given by equation (5). Note that $\pi^{d}(k)$ falls with $k$, and is equal to $\pi^{d}$ when $k=1$. Hence, an increase in $k$ lowers the expected ex post profit per project. The reason for this is that the larger $k$ is, the more severe is the moral hazard in teams problem (the success of each project depends on the joint effort of more agents).

Under the functional structure, each one of the $2 k$ agents specializes in a single task and performs it on the two projects which the firm adopts. The cost that agent $i$ incurs when performing task $i$ on projects 1 and 2 is given by

$$
C^{f}\left(e_{i 1}, e_{i 2}\right)=\gamma e_{i 1}^{2}+\gamma e_{i 2}^{2}-\frac{\sigma e_{i 1} e_{i 2}}{2}, \quad i=1, \ldots 2 k
$$

Using $W_{i j}$ to denote the compensation of agent $i(i=1, \ldots, 2 k)$ if project $j(j=1,2)$ succeeds, and noting that the probability that project $j$ succeeds is $\sum_{i=1}^{2 k} \frac{e_{i j}}{k}$, the contracts offered to the $2 k$ agents are characterized by the solution to the following problem:

$$
\begin{aligned}
& \max \quad \sum_{i=1}^{2 k} \frac{e_{i 1}}{k}\left(R-\sum_{i=1}^{2 k} W_{i 1}\right)+\sum_{i=1}^{2 k} \frac{e_{i 2}}{k}\left(R-\sum_{i=1}^{2 k} W_{i 2}\right) \\
& \left\{e_{i j}, W_{i j}\right\} \\
& \text { s.t. } \quad e_{i 1}, e_{i 2} \in \underset{\widehat{e}_{i 1}, \widehat{e}_{i 2}}{\operatorname{Arg} \max }\left(\frac{\widehat{e}_{i 1}}{k}+\sum_{j \neq i}^{2 k} \frac{e_{j 1}}{k}\right) W_{i 1}+\left(\frac{\widehat{e}_{i 2}}{k}+\sum_{j \neq i}^{2 k} \frac{e_{j 2}}{k}\right) W_{i 2}-C^{f}\left(\widehat{e}_{i 1}, \widehat{e}_{i 2}\right), \quad i=1, \ldots, 2 k, \\
& \left(\sum_{i=1}^{2 k} \frac{e_{i 1}}{k}\right) W_{i 1}+\left(\sum_{i=1}^{2 k} \frac{e_{i 2}}{k}\right) W_{i 2}-C^{f}\left(e_{i 1}, e_{i 2}\right), \quad i=1, \ldots, 2 k .
\end{aligned}
$$


Solving this problem reveals that under the functional structure, the expected ex post profit per project (gross of the cost of investment) is

$$
\pi^{f}(k)=\frac{\pi^{f}}{k^{2}}
$$

where $\pi^{f}$ is given by equation (8). Equation (14) shows that $\pi^{f}(k)$ falls with $k$ but is equal to $\pi^{f}$ when $k=1$. The intuition is that, as in the case of the divisional structure, an increase in $k$ means that the completion of each project requires the joint effort of more agents and hence the moral hazard in teams problem becomes more severe.

Recalling that the managerial moral hazard problem arises only when $\pi^{d}(k)>I$ and $\pi^{f}(k)>I$ (the manager knows that the board will approve costly H-type projects), the fact that $\pi^{d}(k)$ and $\pi^{f}(k)$ are decreasing with $k$ means that an increase in $k$ alleviates the problem under both organizational structures. As before, whenever managerial moral hazard arises only under the divisional structure, i.e., $\pi^{f}(k)<I<\pi^{d}(k)$, the firm is better-off with the divisional structure when $\alpha$ (which reflects the extent of managerial moral hazard) is low, but is better-off with the functional structure when $\alpha$ is high. The critical value of $\alpha$ below which the divisional structure is more profitable ex post is given by $\widehat{\alpha}(k) \equiv \frac{\pi^{d}(k)-I}{\pi^{f}(k)}$. It is easy to see that $\widehat{\alpha}^{\prime}(k)<0$; hence, whenever $\pi^{f}(k)<I<\pi^{d}(k)$, an increase in $k$ favours the functional structure. One should bear in mind though that an increase in $k$ also shrinks the interval for which $\pi^{f}(k)<I<\pi^{d}(k)$.

Proposition 5: An increase in $k$ has the following effects:

(i) It makes the managerial moral hazard problem less severe.

(ii) If $\pi^{f}(k)<I<\pi^{d}(k)$ (only the divisional structure features managerial moral hazard problem), then an increase in $k$ favours the functional structure in the sense the critical value of $\alpha$ beyond which the firm adopts the functional structure decreases. 


\subsection{Asymmetric tasks}

In this subsection we relax the assumption that the two tasks have the same effect on the probability that projects succeed and assume instead that this probability is given by

$$
q\left(e_{p}, e_{m}\right)=\frac{2\left(e_{p}+h e_{m}\right)}{1+h}, \quad h \geq 1
$$

That is, we now assume (without a loss of generality) that task $m$ has a bigger influence on the likelihood of success than task $p$. The rest of the model remains as in Section 2; in particular, the cost that the agents incur when implementing projects is still given by equations (1) and (2). Note that when $h=1$, the likelihood of success is as in Section 2. Moreover, if the efforts are as in Section 3, the likelihood of success and the payoffs are as in Section 3. Hence, the assumption that $h>1$ affects matters only through its effect on the agents' incentives.

Assuming that the firm adopts two identical projects, the expected ex post per-project profit under the divisional structure is given by:

$$
\pi^{d}(h)=\frac{4\left(2 \gamma\left(1+h^{2}\right)+h \rho\right)}{(1+h)^{2}(4 \gamma+\rho)} \pi^{d}
$$

where $\pi^{d}$ is given by (5).

Under the functional structure, one agent specializes in task $p$ and the other specializes in task $m$. But since $h>1$, it is more efficient for the firm to pay agent $m$ an extra dollar at the expense of agent $p$ as the former has a bigger influence on the probability of success. Consequently, at the optimum the firm will set $W_{p 1}=W_{p 2}=0$, and will effectively shut down department $p$. The more productive department $m$, will then perform task $m$ on both projects. ${ }^{9}$ The resulting expected ex post per-project profit is

$$
\pi^{f}(h)=2\left(\frac{h}{1+h}\right)^{2} \pi^{f}
$$

\footnotetext{
${ }^{9}$ Obviously, the result that the firm will only operate department $m$ is an artifact of the assumption that $m$ and $p$ are perfect substitutes. If $m$ and $p$ were imperfect substitutes, then the firm might use both at the optimum, although it would still substitute $p$ for $m$. The main insight though does not depend on whether $m$ and $p$ are perfect or imperfect substitutes.
} 
where $\pi^{f}$ is given by (8).

It is easy to check that $\pi^{d}(1)=\pi^{d}$ and $\pi^{f}(1)=\pi^{f}$, and that both $\pi^{d}(h)$ and $\pi^{f}(h)$ are increasing with $h$. Hence, under both structures, the expected ex post profits increase with the degree of asymmetry between the two tasks. As a result, the managerial moral hazard problem becomes more severe under both structures because the manager will be tempted to recommend costly H-type projects for a larger set of values of $I$. Under the functional structure, $\pi^{f}(h)$ is increasing with $h$ because the firm only operates the $m$ department whose marginal productivity is increasing with $h$. Under the divisional structure, an increase in $h$ induces each agent to shift effort from task $p$ to the more productive task $m$ (the agents however do not quit task $p$ altogether because their cost of effort is quadratic). The reason why $\pi^{d}(h)$ increases with $h$ is that shifting effort from task $m$ to task $p$ has a first order effect on the probability of success and only a second order effect on the cost of effort.

Using equations (15) and (16), it follows that

$$
\frac{\partial}{\partial h}\left(\frac{\pi^{d}(h)}{\pi^{f}(h)}\right)=\frac{-2(4 \gamma+h \rho)(4 \gamma-\sigma)}{h^{3}\left(16 \gamma^{2}-\rho^{2}\right)}<0 .
$$

Hence, an increase in $h$ pushes $\pi^{f}(h)$ closer to $\pi^{d}(h)$. In fact, when $h$ is sufficiently large, $\pi^{f}(h)$ may exceed $\pi^{d}(h)$ provided that

$$
\sigma \geq \widehat{\sigma}(h) \equiv \frac{(4 \gamma+h \rho)^{2}}{2\left(2 \gamma\left(1+h^{2}\right)+h \rho\right)}
$$

Since $\sigma<1$, the functional structure can be ex post more profitable than the divisional structure only when $\widehat{\sigma}(h)<1$. Noting that $\widehat{\sigma}(1)=\frac{4 \gamma+\rho}{2}>1, \widehat{\sigma}^{\prime}(h)<0$, and $\lim _{h \longrightarrow \infty} \widehat{\sigma}(h)=$ $\frac{\rho^{2}}{4 \gamma}<1$, it follows that $\widehat{\sigma}(h)<1$ for sufficiently large values of $h$.

We summarize the discussion in the following proposition:

Proposition 6: An increase in $h$ has the following effects:

(i) It makes the managerial moral hazard problem more severe.

(ii) It favours the functional structure in the sense that it lowers $\frac{\pi^{d}(h)}{\pi^{f}(h)}$; as $h$ becomes 
sufficiently large, $\pi^{f}(h)$ may exceed $\pi^{d}(h)$ provided that there are sufficiently large economies of scale.

Proposition 6 is consistent with Besanko, Régibeau, and Rockett (2005) who show that cross-task asymmetries improve the relative performance of the functional structure. In their model this happens because cross-task asymmetries make it possible to tie the compensation of one functional department more closely to profit than the other. Under the divisional structure by contrast, such differentiation of incentives across tasks is not possible. In our model by contrast, the cross-task asymmetries improve the relative performance of the functional structure because they alleviate the moral hazard in teams problem that arises under this structure.

\section{Narrow business strategies (NBS)}

Another mechanism that might be used to induce the manager to make a better selection of projects is to adopt a narrow business strategy (NBS). Rotemberg and Saloner (1994) show that an NBS allows the firm to induce its employees to exert more effort in generating profitable ideas. Due to contract incompleteness, the firm cannot offer similar incentives when it is involved in many activities. In our case, an NBS commits the firm to focus exclusively on L-type projects and hence solves the managerial moral hazard problem. One way of implementing such NBS is to adopt $\pi^{f}$ as the minimal hurdle rate for project selection. The problem with this policy however is that if the manager recommends H-type projects after all and these projects are profitable, the firm will find it hard to reject his recommendation. Thus, we prefer to think about an NBS as a technological constraint (rather than a policy) that the firm imposes on its ability to implement H-type projects.

To examine the optimal organization design when the firm can adopt an NBS, we will modify our basic setup slightly and assume that with probability $\mu, \mathrm{H}$-type projects require an initial investments $I_{1}$ and with probability $1-\mu$ they require an initial investment $I_{2}$, where $I_{1}<\pi^{f}<I_{2}<\pi^{d}$. As before, L-type projects do not require an initial investment.

When $I=I_{1}$, there is a managerial moral hazard problem under both structures. When 
$I=I_{2}$, the problem arises only under the divisional structure since the manager correctly anticipates that under the functional structure, the board of directors will reject H-type projects.

If the firm adopts an NBS and focuses exclusively on L-type projects, then it will adopt the ex post efficient divisional structure and will implement L-type projects with probability $\alpha$. With probability $1-\alpha$, the firm does not implement any projects. Since L-type projects do not require an initial investment, the expected per-project profit is

$$
O^{N}=\mu \alpha \pi^{d}+(1-\mu) \alpha \pi^{d}=\alpha \pi^{d}
$$

Equation (17) shows that an NBS becomes more profitable as the extent of managerial moral hazard, captured by $\alpha$, increases. The reason for this is simple: an NBS mitigates managerial moral hazard by forcing the firm to adopt only L-type projects. Such projects however are only available with probability $\alpha$. Hence, with probability $1-\alpha$, the firm forgoes H-type projects even if they are profitable. Note that since L-type projects do not require an initial investment, $O^{N}$ is independent of $\mu$.

If the firm does not adopt an NBS, then it needs to choose between the divisional and the functional structures. Under the divisional structure, the firm always adopts H-type projects, so its expected per-project profit is

$$
\begin{aligned}
O^{d} & =\mu\left(\pi^{d}-I_{1}\right)+(1-\mu)\left(\pi^{d}-I_{2}\right) \\
& =\pi^{d}-\left(\mu I_{1}+(1-\mu) I_{2}\right) .
\end{aligned}
$$

Equation (18) shows that the divisional structure is independent of $\alpha$ because it exposes the firm to an extreme form of managerial moral hazard (the manager only recommends H-type projects). Hence, the expected initial investment of each project is $\mu I_{1}+(1-\mu) I_{2}$. However, compared with an NBS, the divisional structure enables the firm to implement projects with probability 1, while under an NBS it implements projects only with probability $\alpha$.

Under a functional structure, the firm implements H-type projects for sure when $I=I_{1}$, but when $I=I_{2}$, it implements only L-type projects, which are available with 
probability $\alpha$. Hence, the firm's expected per-project profit is

$$
\begin{aligned}
O^{f} & =\mu\left(\pi^{f}-I_{1}\right)+(1-\mu) \alpha \pi^{f} \\
& =(\alpha+\mu(1-\alpha)) \pi^{f}-\mu I_{1} .
\end{aligned}
$$

The functional structure then mitigates managerial moral hazard only when the cost of investment is $I_{2}$. With probability $\mu$, the cost of investment is $I_{1}$ and the functional structure fails to solve the managerial moral hazard problem. The firm then implements H-type projects which require an initial investment $I_{1}$. Equation (19) also shows that while the functional structure mitigates the managerial moral hazard problem with probability $1-\mu$, it does poorly with respect to the agents' moral hazard problem: the ex post per-project profit is $\pi^{f}$ rather than $\pi^{d}$. Moreover, under the functional structure, the firm forgoes H-type projects when $I=I_{2}$, so the overall probability that it implements projects is $\alpha+\mu(1-\alpha)$. This probability exceeds $\alpha$ which is the probability that the firm implements projects under NBS, but so long as $\mu<1$, it is below 1 which is the probability that the firm implements projects under the divisional structure. We summarize the discussion in the following proposition.

Proposition 7: Comparing NBS with the divisional and functional structures absent NBS reveals the following:

(i) NBS deals best with managerial moral hazard, followed by the functional structure which deals with the problem only with probability $1-\mu$. The divisional structure fails to mitigate managerial moral hazard. Recalling that $\alpha$ reflects the extent of managerial moral hazard, it follows that NBS is particularly attractive when $\alpha$ is close to 1 , while the divisional structure is particularly attractive when $\alpha$ is close to 0.

(ii) NBS and the divisional structure solve the agents' moral hazard problem and are therefore ex post efficient, while the functional form deals poorly with the agents' moral hazard problem and is ex post inefficient.

(iii) Under the divisional structure, the likelihood that the firm implements projects is 1 , while under the functional structure it is $\alpha+\mu(1-\alpha)$, and under NBS, it is merely $\alpha$. 
To illustrate Proposition 7, suppose that $I_{1}=0.1, I_{2}=0.75, R=2, \gamma=1, \rho=-0.8$, and $\sigma=1$. Then equations (5) and (8) imply that $\pi^{f}=2 / 3$ and $\pi^{d}=5 / 6$. Substituting these numbers in equations (17), (18), and (19), we can present the optimal organizational structure in the following figure for each pair of $\mu$ and $\alpha$ :

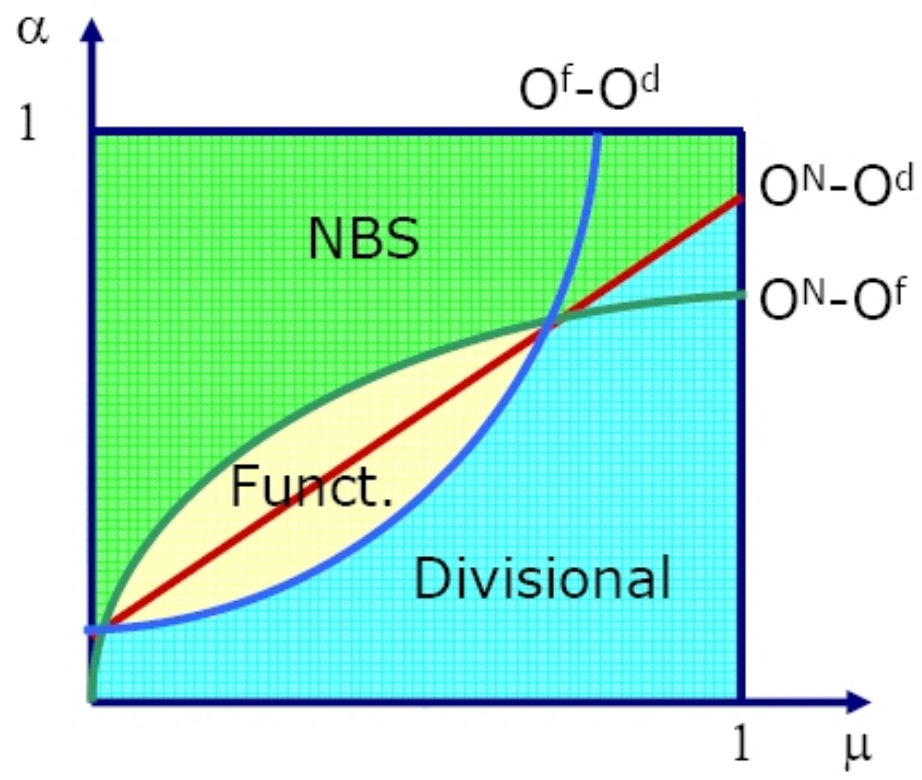

Figure 3: Comparing NBS with the divisional and functional structures absent NBS

An NBS is optimal when $\alpha$ is large, as it deals best with managerial moral hazard. By contrast, the divisional structure, under which the firm only implements H-type projects, is particularly attractive when $\alpha$ is small and when $\mu$, which is the likelihood that H-type projects require a small investment, is high. When $\alpha$ and $\mu$ are intermediate, the functional structure, which deals with the managerial moral hazard problem at least partly, is optimal. Note that an increase in $\mu$ has an ambiguous effect on the optimality of the functional structure, because it makes the firm more susceptible to managerial moral hazard but at the same time it also makes it more likely to implement an H-type project for sure (this happens when $\left.I=I_{1}\right)$ rather than implement an L-type project with probability $\alpha$ (if $I=I_{2}$ ). 


\section{Managerial work overload}

Our theory of organizational structure focuses entirely on horizontal issues: how should the firm allocate the tasks and the projects among its agents? However, the divisional and functional structures also differ from each other with respect to their levels of centralization. Under the functional structure, important decisions are usually taken at the headquarters level, while under the divisional structure they are often delegated to the individual divisions. Some authors like Chandler (1962) and Williamson (1975, 1985), have stressed that the main advantage of the divisional structure over the functional structure is due to this decentralization of decision making which alleviates work overload at the headquarters level and frees up the headquarters' time to focus on long-term issues, such as strategic planning.

Although the selection of projects in our model is done by the manager and the board of directors under both organizational structures, we can nonetheless use our model to explore the effect of managerial work overload for organizational design. To this end, we shall endogenize the choice of $\alpha$ by assuming that the firm can adopt $n$ projects, where $n$ is a positive even integer. The manager receives a private benefit $B$ for each implemented project, regardless of its type, but while he can costlessly discover H-type projects, he needs to exert effort in order to discover L-type projects. Let $\alpha$ be the probability that a project turns out to be L-type, and assume that this probability is independent across projects. The manager's disutility of effort is given by $\psi(n \varphi(\alpha))$, where $\psi(\cdot)$ and $\varphi(\cdot)$ are increasing and convex, and $\varphi^{\prime}(0)=0$ and $\varphi^{\prime}(1)=\infty$.

Given these assumptions, it is clear that if the manager anticipates that the board of directors will accept H-type projects, then he will exert no effort and simply recommend $n$ H-type projects. The manager's utility then is simply $n B$. However, if the manager anticipates that the board of directors will only accept L-type projects, then he will exert effort in order to boost his chances to discover L-type projects. To determine the manager's effort, notice that the discovery of L-type projects follows a binomial distribution since it involves a sequence of $n$ independent trials, each of which either succeeds or fails. Hence, the manager's problem is

$$
\max _{\alpha} n \alpha B-\psi(n \phi(\alpha))
$$


Since $\psi(\cdot)$ and $\varphi(\cdot)$ are increasing and convex and $\varphi^{\prime}(0)=0$ and $\varphi^{\prime}(1)=\infty$, the solution to the manager's problem is $\alpha^{*} \in(0,1)$ and it is determined implicitly by the first order condition:

$$
B=\psi^{\prime}(n \phi(\alpha)) \phi^{\prime}(\alpha)
$$

Since $\psi(\cdot)$ is convex, it follows that $\alpha^{*}$ is decreasing with $n$ : as the firm grows and asks its manager to screen more projects, the likelihood of discovering L-type projects falls.

From earlier results we already know that a decrease in $\alpha$ relaxes the manager's moral hazard problem and therefore favors the ex post efficient divisional structure. Thus, our model supports Williamson's argument that an increase in managerial work overload induces firms to switch from a functional to a divisional structure. In our model, this occurs because managerial work overload diminishes the importance of alleviating managerial moral hazard in the selection of projects.

\section{Conclusion}

In this paper we advance the idea that organizational structure may align the incentive of the firm's manager with those of shareholders. Our main insight is that organizational structures which appear to maximize firm value ex post, may not be optimal once managerial incentives are taken into account: in many cases it is optimal to put in place an organizational structure that appears to be ex post inefficient in order to restrict the management's ability to manipulate investment decisions in the direction it likes.

The idea that the firm may wish to commit itself to an ex post inefficient structure in order to enhance ex ante efficiency is reminiscent of the idea that firms may issue debt which may lead to costly financial distress ex post in order to boost the incentives of their managers ex ante (see e.g., Grossman and Hart, 1982), and the idea that moral hazard in monitoring activity may prevent the refinancing of projects ex post but may improve projects' selection ex ante (Dewatripont and Maskin, 1995). We show that the ex post inefficiency of the functional structure could actually induce the firm's management to improve its selection of projects ex ante. Overall then, the functional structure is likely to be optimal when the managerial moral hazard problem in the selection of projects is sufficiently severe and the 
cost of expensive projects that the management likes is sufficiently high. Otherwise, the firm is better off implementing expensive projects efficiently under the divisional structure.

Our model shows that the organizational structure of the firm may affect the selection of projects and hence the firm's strategy. This implies structure does not follow strategy as Chandler (1962) has argued. Rather, both strategy and structure are jointly determined by more fundamental variables like the firm's technology (economies of scale and scope), managerial ability to identify efficient projects, the cost of projects, and the degree of competitiveness (the profit form successful projects).

\section{References}

Aghion P. and J. Tirole (1995), "Some Implications of Growth for Organizational Form and Ownership Structure," European Economic Review, 39, 440-455.

Alchian A. and H. Demsetz (1972), "Production, Information Costs, and Economic Organization," American Economic Review, 62, 777-795.

Besanko D., P. Régibeau, and K. Rockett (2005), “A Multi-Task Principal-Agent Approach to Organizational Form," Journal of Industrial Economics, 53(4), 437-467.

Chandler A. (1962), Strategy and Structure: Chapter in the History of the American Industrial Enterprise, Cambridge, MA: MIT Press.

Dewatripont M. and E. Maskin (1995), "Credit and Efficiency in Centralized and Decentralized Economies," The Review of Economic Studies, 62(4), 541-555.

Grossman S. and Hart O. (1982), "Corporate Financial Structure and Managerial Incentives," In The Economics of Information and Uncertainty (ed. J. McCall). Chicago: University of Chicago Press.

Harris M. and A. Raviv (2002), "Organizational Design," Management Science, 48(7), $852-865$.

Maskin E., Y. Qian, and C. Xu (2000), "Incentives, Information, and Organizational Form," Review of Economic Studies, 67, 359-378.

Mookherjee D. and M. Tsumagari (2001), "Delegated Management and Contract Intermediation," Mimeo (available at http://econ.bu.edu/dilipm/wkpap.htm/delman.pdf) 
Qian Y., G. Roland, and C. Xu (2006), "Coordination and Experimentation in Mform and U-form Organizations," Journal of Political Economy, 114(2), 336-402.

Rotemberg J. (1999), "Process- versus Function-Based Hierarchies," Journal of Economics and Management Strategy, 8, 453-487.

Rotemberg J. and G. Saloner (1994), "Benefits of Narrow Business Strategies," American Economic Review, 84, 1330-1349.

Williamson H. (1975), Markets and Hierarchies, New York, Free Press.

Williamson H. (1985), The Economic Institutions of Capitalism, New York, Free Press. 